\title{
Erratum to: Quantitative determination of bound water diffusion in multilayer boards by means of neutron imaging
}

\author{
Walter Sonderegger • Stefan Hering • David Mannes • \\ Peter Vontobel • Eberhard Lehmann · Peter Niemz
}

Published online: 29 July 2010

(C) Springer-Verlag 2010

\section{Erratum to: Eur. J. Wood Prod. \\ DOI 10.1007/s00107-010-0463-5}

Due to a processing error the presentation of Table 1 was incorrrect. The unit of $C_{\text {mean.end }}$ was wrongly stated as $\mathrm{kg} / \mathrm{m}^{3}$ instead of $\mathrm{g} / \mathrm{cm}^{3}$. The correct Table 1 is given on next page.

Unfortunately, an error occurred in the German headline to Table 3. The correct German headline is given below.

Tab. 3 Die nach (6)-(9) berechneten Parameter zur Bestimmung der Diffusionskoeffizienten von Holz und Klebstoff. $D_{o . w}, D_{o . a}=$ Diffusionskoeffizienten von Holz bzw. Klebstoff im darrtrockenen Zustand; $\alpha_{w}, \alpha_{a}=$ Konstanten, welche die Feuchteabhängigkeit beschreiben; $\sigma=$ Übergangskoeffizient, $M_{\infty}=$ Feuchtegehalt im Ausgleichszustand; $S=$ Zielfunktionswert

The online version of the original article can be found under doi:10.1007/s00107-010-0463-5.

W. Sonderegger $(\varangle) \cdot$ S. Hering $\cdot$ P. Niemz

Department of Civil, Environmental and Geomatic Engineering, Institute for Building Materials, ETH Zurich, 8093 Zurich,

Switzerland

e-mail: wsonderegger@ethz.ch

D. Mannes · P. Vontobel · E. Lehmann

Spallation Neutron Source (ASQ), Paul Scherrer Institute (PSI),

5232 Villigen, Switzerland 
Table 1 Overview of the tested samples and the applied adhesives at the beginning of the measurements (oven-dry) and the mean moisture content $\left(\mathrm{MC}_{\text {mean.end }}\right)$ and water concentration $\left(C_{\text {mean.end }}\right)$, respectively at the end of the measurements after 70 days (series 1$)$ or 74 days (series 2$)$ exposed to a differentiating climate (series $1: 20^{\circ} \mathrm{C} / 85 \% \mathrm{RH}$ to $0 \% \mathrm{RH}$; series $2: 20^{\circ} \mathrm{C} / 90 \% \mathrm{RH}$ to $0 \% \mathrm{RH}$ )

Tab. 1 Überblick über die untersuchten Proben und die verwendeten Klebstoffe zu Beginn der Messungen (darrtrocken) sowie deren mittlerer Feuchtegehalt $\left(\mathrm{MC}_{\text {mean.end }}\right)$ bzw. Wasserkonzentration $\left(C_{\text {mean.end }}\right)$ am Ende der Messungen, nachdem die Proben 70 Tage (Serie 1) bzw. 74 Tage (Serie 2) einem Differenzklima ausgesetzt worden waren (Serie 1: $20^{\circ} \mathrm{C} / 85 \% \mathrm{rLF}$ zu $0 \% \mathrm{rLF}$; Serie 2: $20{ }^{\circ} \mathrm{C} / 90 \% \mathrm{rLF} \mathrm{zu} 0 \% \mathrm{rLF}$ )

\begin{tabular}{|c|c|c|c|c|c|c|c|c|c|}
\hline & Material/adhesive & No. & $\begin{array}{l}\text { Direction of } \\
\text { diffusion }\end{array}$ & $\begin{array}{l}\text { Bond line } \\
\text { No. } \\
(-)\end{array}$ & $\begin{array}{l}\text { Thickness of } \\
\text { the bond line } \\
(\mathrm{mm})\end{array}$ & $\begin{array}{l}\text { Oven-dry } \\
\text { height } \\
(\mathrm{mm})\end{array}$ & $\begin{array}{l}\text { Oven-dry } \\
\text { density } \\
\left(\mathrm{kg} / \mathrm{m}^{3}\right)\end{array}$ & $\begin{array}{l}\mathrm{MC}_{\text {mean.end }} \\
(\%)\end{array}$ & $\begin{array}{l}C_{\text {mean.end }} \\
\left(\mathrm{g} / \mathrm{cm}^{3}\right)\end{array}$ \\
\hline \multirow[t]{8}{*}{ Series 1} & \multirow[t]{2}{*}{ Spruce wood } & 1 & Tangential & - & - & 29.1 & 418 & 12.8 & 0.054 \\
\hline & & 2 & Radial & - & - & 29.9 & 402 & 12.5 & 0.050 \\
\hline & \multirow[t]{3}{*}{ Urea-1 } & 3 & Tangential & 1 & 0.1 & 29.0 & 398 & 12.5 & 0.050 \\
\hline & & 4 & Tangential & 3 & 0.1 & 29.0 & 441 & 10.6 & 0.047 \\
\hline & & 5 & Tangential & 5 & 0.1 & 29.3 & 457 & 9.6 & 0.044 \\
\hline & \multirow[t]{3}{*}{ PUR-1 } & 6 & Tangential & 1 & 0.1 & 29.0 & 403 & 12.0 & 0.048 \\
\hline & & 7 & Tangential & 3 & 0.1 & 29.3 & 430 & 7.9 & 0.034 \\
\hline & & 8 & Tangential & 5 & 0.1 & 29.7 & 476 & 6.1 & 0.029 \\
\hline \multirow[t]{12}{*}{ Series 2} & \multirow[t]{3}{*}{ Epoxy } & 9 & Tangential & 1 & 0.1 & 29.3 & 429 & 14.0 & 0.060 \\
\hline & & 10 & Tangential & 1 & 0.5 & 29.5 & 586 & 11.0 & 0.064 \\
\hline & & 11 & Tangential & 1 & 1.0 & 30.1 & 523 & 11.0 & 0.058 \\
\hline & \multirow[t]{3}{*}{ PVAc } & 12 & Tangential & 1 & 0.1 & 29.3 & 407 & 14.8 & 0.060 \\
\hline & & 13 & Tangential & 1 & 0.5 & 29.5 & 389 & 15.3 & 0.060 \\
\hline & & 14 & Tangential & 1 & 1.0 & 29.8 & 351 & 14.6 & 0.051 \\
\hline & \multirow[t]{3}{*}{ Urea-2 } & 15 & Tangential & 1 & 0.1 & 29.5 & 420 & 15.0 & 0.063 \\
\hline & & 16 & Tangential & 1 & 0.5 & 29.5 & 419 & 15.3 & 0.064 \\
\hline & & 17 & Tangential & 1 & 1.0 & 29.8 & 417 & 14.9 & 0.062 \\
\hline & \multirow[t]{3}{*}{ PUR-2 } & 18 & Tangential & 1 & 0.1 & 29.4 & 458 & 14.2 & 0.065 \\
\hline & & 19 & Tangential & 1 & 0.5 & 29.8 & 403 & 13.5 & 0.054 \\
\hline & & 20 & Tangential & 1 & 1.0 & 30.5 & 452 & 12.4 & 0.056 \\
\hline
\end{tabular}

